PROCEEDINGS OF THE

AMERICAN MATHEMATICAL SOCIETY

Volume 138, Number 10, October 2010, Pages 3501-3507

S 0002-9939(2010)10395-7

Article electronically published on May 14, 2010

\title{
CONSTANTIVE MAL'CEV CLONES ON FINITE SETS ARE FINITELY RELATED
}

\author{
ERHARD AICHINGER
}

(Communicated by Julia Knight)

\begin{abstract}
We show that on a finite set there are at most countably many constantive clones that contain a Mal'cev operation, and each such clone can be described by a single finitary relation. Thus, modulo polynomial equivalence and renaming of the elements, there are only countably many finite algebras that contain a Mal'cev term.
\end{abstract}

\section{INTRODUCTION}

Following [8], an algebra $\mathbf{A}$ is a pair $\langle A, F\rangle$, where $A$ is a non-empty set and $F$, the set of fundamental operations of $A$, is a subset of $O(A):=\bigcup\left\{A^{A^{n}} \mid n \in \mathbb{N}_{0}\right\}$, the set of finitary operations on $A$. It is clear that for a finite set $A$ with $|A| \geq 2$, $O(A)$ has $2^{\aleph_{0}}$ subsets, and hence there are $2^{\aleph_{0}}$ different algebras on $A$. However, we will take the view that two algebras can be considered equivalent if the same operations can be built from their fundamental operations. One concept to describe such an equivalence is the notion of polynomial equivalence from universal algebra 14. In particular, two algebras $\mathbf{A}_{1}=\langle A, F\rangle$ and $\mathbf{A}_{2}=\langle A, G\rangle$ defined on the same base set are polynomially equivalent if they have the same clone of polynomial operations. On a two-element set, there are exactly 7 polynomially inequivalent algebras. However, by [1], if $A$ is finite and $|A| \geq 3$, then there are $2^{\aleph_{0}}$ polynomially inequivalent algebras on $A$. In this paper, we restrict our attention to algebras with a Mal'cev term (Mal'cev algebras for short). By [6, Theorem 12.2] these are exactly the algebras that generate congruence permutable varieties. Among those algebras are all finite algebras that have a quasigroup operation among their binary term functions, and all groups and rings. In 9, P. Idziak proved that on a finite set $A$, the number of polynomially inequivalent Mal'cev algebras is finite if and only if $|A| \leq 3$, and he asks whether this number can be uncountable for a finite $A$ (cf. [5, Problem 8]). For certain algebras, it is known that they have only finitely many polynomially inequivalent expansions. Among such algebras are all groups of squarefree order [11] and, using results on polynomial completeness [10], for example, all finite Mal'cev algebras omitting type $\mathbf{2}$ in the sense of Tame Congruence Theory 8 .

These questions have also often been stated using the language of clone theory [16, 17, 14. A clone on a set $A$ is a set of finitary operations on $A$ that contains all projection operations $p\left(x_{1}, \ldots, x_{n}\right):=x_{i}(n, i \in \mathbb{N}, i \leq n)$ and is closed under

Received by the editors August 24, 2009 and, in revised form, January 19, 2010.

2010 Mathematics Subject Classification. Primary 08A40.

(C) 2010 American Mathematical Society
Reverts to public domain 28 years from publication 3501 
all compositions. It is called constantive if it contains all constant operations, and it is called a Mal'cev clone if it contains a ternary operation satisfying $d(a, a, b)=$ $d(b, a, a)=b$ for all $a, b \in A$. Constantive Mal'cev clones are exactly those sets that arise as the sets of polynomial operations of some Mal'cev algebra; therefore, often "polynomial clone" is used instead of "constantive clone". Now, the exact wording of Problem 8 in [5] is: Does there exist a finite set with uncountably many polynomial Mal'cev clones? Such a set cannot exist if every constantive Mal'cev clone on a finite set has some "finite representation". It follows from 9] that there are constantive Mal'cev clones that cannot be generated by finitely many operations. However, 16] provides a representation of clones on finite sets through their finitary invariant relations, and it has indeed been shown for many classes of constantive Mal'cev clones that they can be described by finitely many of these relations (they are, in short, finitely related). For example, the clone of polynomial functions has been shown to be finitely related for the following algebras: all finite groups, all of whose Sylow subgroups are abelian, and all finite commutative rings with 1 12; all Mal'cev algebras with congruence lattice of height at most 2 and all supernilpotent Mal'cev algebras [2] and all expansions of groups of order $p^{2}(p$ a prime) [4. Many of the results in these papers provide concrete bounds for the arity of the relations that determine polynomials.

In 2006, P. Idziak expressed the conjecture that every constantive Mal'cev clone is finitely related. In the present paper, we prove this conjecture by showing that there is no infinite descending chain of constantive Mal'cev clones on a finite set. Hence, every constantive Mal'cev clone is finitely related, and therefore, on a finite set, there exist at most $\aleph_{0}$ constantive Mal'cev clones. This also provides a partial answer to Problem 5.6 in [13], which asks whether there exists a Mal'cev operation on a finite set that is contained in uncountably many clones.

\section{Preliminaries From ORDER THEORY}

Let $\mathbb{A}=\langle A, \leq\rangle$ be a partially ordered set. We say that $\mathbb{A}$ satisfies the descending chain condition (DCC) if there is no infinite descending chain $a_{1}>a_{2}>a_{3}>\ldots$; $\mathbb{A}$ satisfies the ascending chain condition (ACC) if there is no infinite ascending chain $a_{1}<a_{2}<a_{3}<\ldots$. A subset $I$ of $A$ is an upward closed subset if for all $a \in I$ and $b \in A$ with $a \leq b$, we have $b \in I$. The set of all upward closed subsets of $\mathbb{A}$ is denoted by $\mathcal{U}(\mathbb{A})$.

For a set $B$, let $B^{+}:=\bigcup\left\{B^{n} \mid n \in \mathbb{N}\right\}$ be the set of all words over the alphabet $B$. For $\mathbf{x}=\left(x_{1}, \ldots, x_{m}\right)$ and $\mathbf{y}=\left(y_{1}, \ldots, y_{n}\right)$ in $B^{+}$, we say that $\mathbf{x}$ can be embedded into $\mathbf{y}$, denoted by $\mathbf{x} \leq_{e} \mathbf{y}$, if there are $i_{1}<i_{2}<\ldots<i_{m} \in\{1, \ldots, n\}$ such that

$\left(y_{1}, \ldots, y_{n}\right)=\left(y_{1}, \ldots, y_{i_{1}-1}, x_{1}, y_{i_{1}+1}, \ldots, y_{i_{2}-1}, x_{2}, y_{i_{2}+1}, \ldots, x_{m}, y_{i_{m}+1}, \ldots, y_{n}\right)$.

We will use the following fact (Higman's Theorem) about this ordering.

Proposition 2.1. Let $B$ be a finite set. Then $\left\langle B^{+}, \leq_{e}\right\rangle$ has no infinite antichain, and $\left\langle\mathcal{U}\left(\left\langle B^{+}, \leq_{e}\right\rangle\right), \subseteq\right\rangle$ satisfies the $(\mathrm{ACC})$.

The fact that $\left\langle B^{+}, \leq_{e}\right\rangle$ has no infinite antichain is Theorem 4.4 of [7]. The partially ordered set $\left\langle B^{+}, \leq_{e}\right\rangle$ obviously satisfies the (DCC), and so the second part follows from the theory of well-quasi-orderings ([15, Theorem 1.2]; cf. [3, Proposition 3.1]).

For a partially ordered set $\mathbb{A}$ and $m \in \mathbb{N}$, we define a partially ordered set $\mathbb{A}^{m}=\left\langle A^{m}, \leq\right\rangle$, where $\left(a_{1}, \ldots, a_{m}\right) \leq\left(b_{1}, \ldots, b_{m}\right)$ if for all $i \in\{1, \ldots, m\}$, we 
have $a_{i} \leq b_{i}$. From Proposition 2.1 it follows immediately that for every $m \in \mathbb{N}$, $\left\langle\mathcal{U}\left(\left\langle B^{+}, \leq_{e}\right\rangle\right), \subseteq\right\rangle^{m}$ satisfies the (ACC).

\section{MaL'Cev algebras}

For the notions of algebra and clone, we refer to the introductory Chapter 0 of 8. A ternary operation $d$ on a set $A$ is called a Mal'cev operation if $d(a, a, b)=$ $d(b, a, a)=b$ for all $a, b \in A$. We call an algebra a Mal'cev algebra if it has a Mal'cev operation among its ternary term functions. A clone is called a Mal'cev clone if it has a Mal'cev operation among its ternary operations. A clone on the set $A$ is called constantive if it contains all unary constant operations on $A$. For a clone $\mathbf{C}$ and $n \in \mathbb{N}$, we denote the set of its $n$-ary operations by $\mathbf{C}^{[n]}$.

Let $\mathbf{A}$ be a Mal'cev algebra, let $m \in \mathbb{N}$, and let $F$ be a subuniverse of $\mathbf{A}^{m}$. For $i \in\{1, \ldots, m\}$, we define the relation $\varphi_{i}(F)$ on $A$ by

$\varphi_{i}(F):=\left\{\left(a_{i}, b_{i}\right) \mid\left(a_{1}, \ldots, a_{m}\right) \in F,\left(b_{1}, \ldots, b_{m}\right) \in F,\left(a_{1}, \ldots, a_{i-1}\right)=\left(b_{1}, \ldots, b_{i-1}\right)\right\}$.

Lemma 3.1. Let $\mathbf{A}$ be a Mal'cev algebra with Mal'cev term $d$, and let $m \in \mathbb{N}$. Let $F, G$ be subuniverses of $\mathbf{A}^{m}$ with $F \subseteq G$. We assume that for all $i \in\{1, \ldots, m\}$, we have $\varphi_{i}(G) \subseteq \varphi_{i}(F)$. Then $F=G$.

Proof. For each $k \in\{1, \ldots, m\}$, let

$$
\begin{aligned}
F_{k} & :=\left\{\left(f_{1}, \ldots, f_{k}\right) \mid\left(f_{1}, \ldots, f_{m}\right) \in F\right\}, \\
G_{k} & :=\left\{\left(g_{1}, \ldots, g_{k}\right) \mid\left(g_{1}, \ldots, g_{m}\right) \in G\right\} .
\end{aligned}
$$

We will now prove by induction on $k$ that for all $k \in\{1, \ldots, m\}$, we have $G_{k} \subseteq$ $F_{k}$. For $k=1$, let $\left(g_{1}\right) \in G_{1}$. Then we have $\left(g_{1}, g_{1}\right) \in \varphi_{1}(G)$, and thus, by assumption, $\left(g_{1}, g_{1}\right) \in \varphi_{1}(F)$. This implies that there is $\left(f_{1}, \ldots, f_{m}\right) \in F$ with $f_{1}=g_{1}$. Therefore $\left(g_{1}\right) \in F_{1}$.

Now let $k \geq 2$. We fix $\left(g_{1}, \ldots, g_{k}\right) \in G_{k}$. Then there is $\left(g_{k+1}, \ldots, g_{m}\right) \in$ $A^{m-k}$ such that $\left(g_{1}, \ldots, g_{m}\right) \in G$. Thus, $\left(g_{1}, \ldots, g_{k-1}\right) \in G_{k-1}$. By the induction hypothesis, we obtain $\left(g_{1}, \ldots, g_{k-1}\right) \in F_{k-1}$. Thus there is $\left(f_{1}, \ldots, f_{m}\right) \in F$ such that $f_{1}=g_{1}, f_{2}=g_{2}, \ldots, f_{k-1}=g_{k-1}$. Since $F \subseteq G$, we have $\left(f_{1}, \ldots, f_{m}\right) \in G$. Therefore, we have $\left(f_{k}, g_{k}\right) \in \varphi_{k}(G)$. By the assumptions, we have $\left(f_{k}, g_{k}\right) \in$ $\varphi_{k}(F)$. Hence there are $a_{1}, \ldots, a_{k-1} \in A$ such that $\left(a_{1}, \ldots, a_{k-1}, f_{k}\right) \in F_{k}$ and $\left(a_{1}, \ldots, a_{k-1}, g_{k}\right) \in F_{k}$. Thus, we have

$$
d\left(\left(f_{1}, \ldots, f_{k-1}, f_{k}\right),\left(a_{1}, \ldots, a_{k-1}, f_{k}\right),\left(a_{1}, \ldots, a_{k-1}, g_{k}\right)\right) \in F_{k} .
$$

Hence $\left(f_{1}, \ldots, f_{k-1}, g_{k}\right) \in F_{k}$, and thus $\left(g_{1}, \ldots, g_{k-1}, g_{k}\right) \in F_{k}$. This completes the induction step.

Therefore, we have $G_{m} \subseteq F_{m}$, which means $G \subseteq F$.

Let $b \in \mathbb{N}$, and let $A:=\{1, \ldots, b\}$. For $n \in \mathbb{N}$, we use the lexicographic ordering $\leq_{\text {lex }}$ on $A^{n}$ that is defined by

$$
\begin{aligned}
& \left(x_{1}, \ldots, x_{n}\right) \leq_{\operatorname{lex}}\left(y_{1}, \ldots, y_{n}\right): \Leftrightarrow \\
& \quad\left(\exists i \in\{1, \ldots, n\}: x_{1}=y_{1} \wedge \ldots \wedge x_{i-1}=y_{i-1} \wedge x_{i}<y_{i}\right) \text { or } \\
& \quad\left(x_{1}, \ldots, x_{n}\right)=\left(y_{1}, \ldots, y_{n}\right) .
\end{aligned}
$$




\section{Constantive clones}

Let $\mathbf{C}$ be a clone on $A=\{1, \ldots, b\}$. For $n \in \mathbb{N}$ and $\mathbf{x} \in A^{n}$, we define a binary relation $\varphi(\mathbf{C}, \mathbf{x})$ on $A$ by

$$
\begin{aligned}
\varphi(\mathbf{C}, \mathbf{x}):=\left\{(f(\mathbf{x}), g(\mathbf{x})) \mid f, g \in \mathbf{C}^{[n]},\right. & \\
& \left.\forall \mathbf{z} \in A^{n}:\left(\mathbf{z} \leq_{\text {lex }} \mathbf{x} \text { and } \mathbf{z} \neq \mathbf{x}\right) \Rightarrow f(\mathbf{z})=g(\mathbf{z})\right\} .
\end{aligned}
$$

Lemma 4.1. Let $b, m, n \in \mathbb{N}$, let $\mathbf{C}$ be a constantive clone on $A=\{1, \ldots, b\}$, and let $\mathbf{x} \in A^{m}, \mathbf{y} \in A^{n}$ such that $\mathbf{x} \leq_{e} \mathbf{y}$. Then $\varphi(\mathbf{C}, \mathbf{y}) \subseteq \varphi(\mathbf{C}, \mathbf{x})$.

Proof. Let $(a, b) \in \varphi(\mathbf{C}, \mathbf{y})$. Then there are $f, g \in \mathbf{C}^{[n]}$ such that $a=f(\mathbf{y})$, $b=g(\mathbf{y})$, and $f(\mathbf{z})=g(\mathbf{z})$ for all $\mathbf{z} \in A^{n}$ with $\mathbf{z} \leq_{\text {lex }} \mathbf{y}, \mathbf{z} \neq \mathbf{y}$. Since $\mathbf{x} \leq_{e} \mathbf{y}$, there are $i_{1}<i_{2}<\ldots<i_{m} \in\{1, \ldots, n\}$ such that

$$
\left(y_{1}, \ldots, y_{n}\right)=\left(y_{1}, \ldots, y_{i_{1}-1}, x_{1}, y_{i_{1}+1}, \ldots, y_{i_{2}-1}, x_{2}, y_{i_{2}+1}, \ldots, x_{m}, y_{i_{m}+1}, \ldots, y_{n}\right) .
$$

Now we define functions $f_{1}$ and $g_{1}$ from $A^{m}$ to $A$ by

$$
\begin{aligned}
& f_{1}\left(\xi_{1}, \ldots, \xi_{m}\right):= \\
& f\left(y_{1}, \ldots, y_{i_{1}-1}, \xi_{1}, y_{i_{1}+1}, \ldots, y_{i_{2}-1}, \xi_{2}, y_{i_{2}+1}, \ldots, \xi_{m}, y_{i_{m}+1}, \ldots, y_{n}\right), \\
& g_{1}\left(\xi_{1}, \ldots, \xi_{m}\right):= \\
& g\left(y_{1}, \ldots, y_{i_{1}-1}, \xi_{1}, y_{i_{1}+1}, \ldots, y_{i_{2}-1}, \xi_{2}, y_{i_{2}+1}, \ldots, \xi_{m}, y_{i_{m}+1}, \ldots, y_{n}\right)
\end{aligned}
$$

for $\xi_{1}, \ldots, \xi_{m} \in A$. Since $\mathbf{C}$ is constantive, both $f_{1}$ and $g_{1}$ are elements of $\mathbf{C}$.

We will now show that $\left(f_{1}(\mathbf{x}), g_{1}(\mathbf{x})\right)$ is an element of $\boldsymbol{\varphi}(\mathbf{C}, \mathbf{x})$. To this end, let $\mathbf{z} \in A^{m}$ be such that $\mathbf{z} \leq_{\text {lex }} \mathbf{x}, \mathbf{z} \neq \mathbf{x}$. Then we have

$$
f_{1}(\mathbf{z})=f\left(y_{1}, \ldots, y_{i_{1}-1}, z_{1}, y_{i_{1}+1}, \ldots, y_{i_{2}-1}, z_{2}, y_{i_{2}+1}, \ldots, z_{m}, y_{i_{m}+1}, \ldots, y_{n}\right) .
$$

Since $\mathbf{z} \leq_{\text {lex }} \mathbf{x}$, we see that

$\left(y_{1}, \ldots, y_{i_{1}-1}, z_{1}, y_{i_{1}+1}, \ldots, y_{i_{2}-1}, z_{2}, y_{i_{2}+1}, \ldots, z_{m}, y_{i_{m}+1}, \ldots, y_{n}\right) \leq_{\text {lex }}\left(y_{1}, \ldots, y_{n}\right)$.

Hence, since $\mathbf{z} \neq \mathbf{x}$, we have

$$
\begin{aligned}
& f\left(y_{1}, \ldots, y_{i_{1}-1}, z_{1}, y_{i_{1}+1}, \ldots, y_{i_{2}-1}, z_{2}, y_{i_{2}+1}, \ldots, z_{m}, y_{i_{m}+1}, \ldots, y_{n}\right) \\
& =g\left(y_{1}, \ldots, y_{i_{1}-1}, z_{1}, y_{i_{1}+1}, \ldots, y_{i_{2}-1}, z_{2}, y_{i_{2}+1}, \ldots, z_{m}, y_{i_{m}+1}, \ldots, y_{n}\right)=g_{1}(\mathbf{z}) .
\end{aligned}
$$

From this, we obtain $\left(f_{1}(\mathbf{x}), g_{1}(\mathbf{x})\right) \in \varphi(\mathbf{C}, \mathbf{x})$. Since $\left(f_{1}(\mathbf{x}), g_{1}(\mathbf{x})\right)=(f(\mathbf{y}), g(\mathbf{y}))=$ $(a, b)$, we obtain $(a, b) \in \varphi(\mathbf{C}, \mathbf{x})$.

Definition 4.2. Let $b \in \mathbb{N}$, let $\mathbf{C}$ be a clone on $A=\{1, \ldots, b\}$, and let $\alpha \subseteq A \times A$. We define a subset $\Psi(\mathbf{C}, \alpha)$ of $A^{+}$by $\Psi(\mathbf{C}, \alpha):=\left\{\mathbf{x} \in A^{+} \mid \boldsymbol{\varphi}(\mathbf{C}, \mathbf{x}) \subseteq \alpha\right\}$.

Lemma 4.3. Let $b \in \mathbb{N}$, let $\mathbf{C}$ be a constantive clone on $A=\{1, \ldots, b\}$, and let $\alpha \subseteq A \times A$. Then $\Psi(\mathbf{C}, \alpha)$ is an upward closed subset of $\left\langle A^{+}, \leq_{e}\right\rangle$.

Proof. Let $\mathbf{x} \in \Psi(\mathbf{C}, \alpha)$, and let $\mathbf{y} \in A^{+}$such that $\mathbf{x} \leq_{e} \mathbf{y}$. Since $\mathbf{x} \in \Psi(\mathbf{C}, \alpha)$, we have $\varphi(\mathbf{C}, \mathbf{x}) \subseteq \alpha$. By Lemma 4.1 we have $\varphi(\mathbf{C}, \mathbf{y}) \subseteq \varphi(\mathbf{C}, \mathbf{x})$. Therefore, $\boldsymbol{\varphi}(\mathbf{C}, \mathbf{y}) \subseteq \alpha$, and thus $\mathbf{y} \in \Psi(\mathbf{C}, \alpha)$. 


\section{Chains of Constantive Mal'CeV Clones}

Lemma 5.1. Let $b \in \mathbb{N}$, and let $A:=\{1, \ldots, b\}$. Let $(\mathcal{C}, \subseteq)$ be a linearly ordered set of constantive clones on A such that all contain the same Mal'cev operation d. Let $\mathbf{C}, \mathbf{D} \in \mathcal{C}$. Then the following are equivalent:

(1) $\mathbf{C} \subseteq \mathbf{D}$.

(2) For all $\alpha \subseteq A \times A$, we have $\Psi(\mathbf{D}, \alpha) \subseteq \Psi(\mathbf{C}, \alpha)$.

Proof. (11) $\Rightarrow(2)$ : Let $\alpha \subseteq A \times A$, and let $\mathbf{x} \in \Psi(\mathbf{D}, \alpha)$. Then $\varphi(\mathbf{D}, \mathbf{x}) \subseteq \alpha$. Since $\mathbf{C} \subseteq \mathbf{D}$, we therefore have $\boldsymbol{\varphi}(\mathbf{C}, \mathbf{x}) \subseteq \alpha$, and thus $\mathbf{x} \in \Psi(\mathbf{C}, \alpha)$. (2) $\Rightarrow(\mathbb{1})$ : Since $\mathcal{C}$ is a linearly ordered set of clones, we either have $\mathbf{C} \subseteq \mathbf{D}$ or $\mathbf{D} \subseteq \mathbf{C}$. In the first case, there is nothing to prove, so we assume $\mathbf{D} \subseteq \mathbf{C}$.

We will now prove that in this case, we have $\mathbf{C}^{[n]} \subseteq \mathbf{D}^{[n]}$ for all $n \in \mathbb{N}$. To this end, we let $n \in \mathbb{N}$ and show that for all $\mathbf{x} \in A^{n}$,

$$
\varphi(\mathbf{C}, \mathbf{x}) \subseteq \varphi(\mathbf{D}, \mathbf{x}) .
$$

In order to prove (5.1), we fix $\mathbf{x} \in A^{n}$. We obviously have $\mathbf{x} \in \Psi(\mathbf{D}, \varphi(\mathbf{D}, \mathbf{x}))$. By the assumption (2) , we therefore have $\mathbf{x} \in \Psi(\mathbf{C}, \boldsymbol{\varphi}(\mathbf{D}, \mathbf{x}))$. Hence $\varphi(\mathbf{C}, \mathbf{x}) \subseteq$ $\varphi(\mathbf{D}, \mathbf{x})$, which concludes the proof of (5.1).

Now $F:=\mathbf{D}^{[n]}$ and $G:=\mathbf{C}^{[n]}$ are subuniverses of $\langle A, d\rangle^{A^{n}}$. Hence from (5.1) and Lemma 3.1 (with $m:=|A|^{n}$ ), we obtain $\mathbf{C}^{[n]}=\mathbf{D}^{[n]}$.

Let $2^{A \times A}$ denote the power set of $A \times A$. On the set $\mathbb{U}:=\mathcal{U}\left(\left\langle A^{+}, \leq_{e}\right\rangle\right)^{2^{A \times A}}$, we define an order as follows: for $\mathbf{S}=\langle S(\alpha) \mid \alpha \subseteq A \times A\rangle$ and $\mathbf{T}=\langle T(\alpha)| \alpha \subseteq$ $A \times A\rangle \in \mathbb{U}$, we define $\mathbf{S} \leq \mathbf{T}$ to mean that $S(\alpha) \subseteq T(\alpha)$ for all $\alpha \subseteq A \times A$. Under this ordering, $\langle\mathbb{U}, \leq\rangle$ is isomorphic to the $2^{|A|^{2}}$-fold direct product of $\left\langle\mathcal{U}\left(\left\langle A^{+}, \leq_{e}\right\rangle\right), \subseteq\right\rangle$. Therefore, it follows from Proposition 2.1 that $\langle\mathbb{U}, \leq\rangle$ satisfies the (ACC).

Now, as a corollary of Lemma 5.1, we obtain:

Lemma 5.2. Let $b \in \mathbb{N}$, and let $A:=\{1, \ldots, b\}$. Let $(\mathcal{C}, \subseteq)$ be a linearly ordered set of constantive clones on A such that all contain the same Mal'cev operation d, and let $\mathbb{U}:=\mathcal{U}\left(\left\langle A^{+}, \leq_{e}\right\rangle\right)^{2^{A \times A}}$. Let $R: \mathcal{C} \rightarrow \mathbb{U}$ be defined by

$$
R(\mathbf{C}):=\langle\Psi(\mathbf{C}, \alpha) \mid \alpha \subseteq A \times A\rangle
$$

for $\mathbf{C} \in \mathcal{C}$. Then $R$ is injective, and for all $\mathbf{C}, \mathbf{D} \in \mathcal{C}$ with $\mathbf{C} \subseteq \mathbf{D}$, we have $R(\mathbf{D}) \leq R(\mathbf{C})$.

For a finite set $A$ and a set $R$ of finitary relations on $A$, we will write $\operatorname{Pol}(A, R)$ for the set of those functions on $A$ that preserve all relations in $R$ (cf. [16]).

Theorem 5.3. Let $A$ be a finite set, and let $\mathcal{M}$ be the set of all constantive Mal'cev clones on $A$. Then we have:

(1) There is no infinite descending chain in $(\mathcal{M}, \subseteq)$.

(2) For every constantive Mal'cev clone $\mathbf{C}$ on $A$, there is a finitary relation $\rho$ on $A$ such that $\mathbf{C}=\operatorname{Pol}(A,\{\rho\})$.

(3) The set $\mathcal{M}$ is finite or countably infinite.

Proof. (11) Let $\mathcal{C}$ be an infinite descending chain of constantive Mal'cev clones on the finite set $A$. Since there are only finitely many Mal'cev operations on a finite set, infinitely many clones in $\mathcal{C}$ have the same Mal'cev operation $d$. From these clones, Lemma 5.2 produces an infinite ascending chain in $\left\langle\mathcal{U}\left(\left\langle A^{+}, \leq_{e}\right\rangle\right), \subseteq\right\rangle^{2^{A \times A}}$, which contradicts Proposition 2.1. 
(2) Let $\mathbf{C}$ be a constantive Mal'cev clone on the finite set $A$. Using the implication (ii) $\Rightarrow(\text { i })^{\prime}$ in [16, Charakterisierungssatz 4.1.3], it follows from (11) that there is a finite set $R$ of finitary relations on $A$ such that $\mathbf{C}=\operatorname{Pol}(A, R)$. By [16, p. 50], there is a single finitary relation $\rho$ on $A$ with $\operatorname{Pol}(A, R)=\operatorname{Pol}(A,\{\rho\})$.

(3) Every finitary relation on the finite set $A$ is a finite subset of the countable set $A^{+}$. Hence the claim follows from (2).

Using [9], we obtain that the number of constantive Mal'cev clones on a finite set $A$ is finite if $|A| \leq 3$, and $\aleph_{0}$ for $|A| \geq 4$.

\section{ACKNOWLEDGMENTS}

P. Idziak and R. McKenzie have drawn the author's attention to the question considered here. The author is grateful to them, and to J. Farley, P. Mayr, and N. Mudrinski for helpful discussions.

\section{REFERENCES}

1. I. Ágoston, J. Demetrovics, and L. Hannák, On the number of clones containing all constants (a problem of R. McKenzie), Lectures in universal algebra (Szeged, 1983), Colloq. Math. Soc. János Bolyai, vol. 43, North-Holland, Amsterdam, 1986, pp. 21-25.

2. E. Aichinger and N. Mudrinski, Polynomial clones of Mal'cev algebras with small congruence lattices, Acta Math. Hungar. 124 (2010), no. 4, 315-333.

3. M. Aschenbrenner and R. Hemmecke, Finiteness theorems in stochastic integer programming, Found. Comput. Math. 7 (2007), no. 2, 183-227. MR2324416 (2008c:90031)

4. A. A. Bulatov, Polynomial clones containing the Mal'tsev operation of the groups $\mathbb{Z}_{p^{2}}$ and $\mathbb{Z}_{p} \times \mathbb{Z}_{p}$, Mult.-Valued Log. 8 (2002), no. 2, 193-221. MR1957653(2004a:08001)

5. A. A. Bulatov and P. M. Idziak, Counting Mal'tsev clones on small sets, Discrete Math. 268 (2003), no. 1-3, 59-80. MR1982389 (2004i:08003)

6. S. Burris and H. P. Sankappanavar, A course in universal algebra, Springer, New YorkHeidelberg-Berlin, 1981. MR648287 (83k:08001)

7. G. Higman, Ordering by divisibility in abstract algebras, Proc. London Math. Soc. (3) 2 (1952), 326-336. MR0049867 (14:238e)

8. D. Hobby and R. McKenzie, The structure of finite algebras, Contemporary Mathematics, vol. 76, American Mathematical Society, 1988. MR958685 (89m:08001)

9. P. M. Idziak, Clones containing Mal'tsev operations, Internat. J. Algebra Comput. 9 (1999), no. 2, 213-226. MR.1703074 (2000e:08007)

10. K. Kaarli and A. F. Pixley, Polynomial completeness in algebraic systems, Chapman \& Hall/CRC, Boca Raton, Florida, 2001. MR.1888967 (2003a:08001)

11. P. Mayr, Polynomial clones on squarefree groups, Internat. J. Algebra Comput. 18 (2008), no. 4, 759-777. MR2428154 (2009f:08003)

12. _ Mal'cev algebras with supernilpotent centralizers, Algebra Universalis, accepted for publication.

13. R. McKenzie, K. Kearnes, E. Kiss, and A. Szendrei, Sixty-four problems in universal algebra, http://www.math.u-szeged.hu/confer/algebra/2001/progr.html, 2001, Problems posed at "A course in tame congruence theory", Paul Erdős Summer Research Center, Budapest, in July 2001.

14. R. N. McKenzie, G. F. McNulty, and W. F. Taylor, Algebras, lattices, varieties, volume I, Wadsworth \& Brooks/Cole Advanced Books \& Software, Monterey, California, 1987. MR.883644 (88e:08001)

15. E. C. Milner, Basic wqo- and bqo-theory, Graphs and order (Banff, Alta., 1984), NATO Adv. Sci. Inst. Ser. C Math. Phys. Sci., vol. 147, Reidel, Dordrecht, 1985, pp. 487-502. MR818505 (87h:04004) 
16. R. Pöschel and L. A. Kalužnin, Funktionen- und Relationenalgebren, Mathematische Monographien [Mathematical Monographs], vol. 15, VEB Deutscher Verlag der Wissenschaften, Berlin, 1979, Ein Kapitel der diskreten Mathematik [A chapter in discrete mathematics]. MR.543839 (81f:03075)

17. Á. Szendrei, Clones in universal algebra, Séminaire de Mathématiques Supérieures [Seminar on Higher Mathematics], vol. 99, Presses de l'Université de Montréal, Montreal, QC, 1986. MR859550(87m:08005)

Institut für Algebra, Johannes Kepler Universität Linz, Altenbergerstrasse 69, 4040 Linz, AUSTRIA

E-mail address: erhard@algebra.uni-linz.ac.at 